\title{
(2) OPEN ACCESS \\ Perceived effectiveness of four different cigarette health warning label themes among a sample of urban smokers and non-smokers in China
}

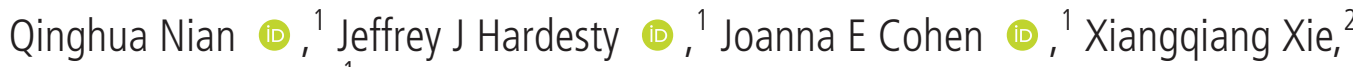 \\ Ryan David Kennedy ${ }^{1}$
}

\begin{abstract}
- Additional supplemental material is published online only. To view, please visit the journal online (http://dx.doi. org/10.1136/tobaccocontrol2021-056703).
\end{abstract}

${ }^{1}$ Institute for Global Tobacco Control, Department of Health, Behavior and Society, Johns Hopkins Bloomberg School of Public Health, Baltimore, MD, USA

${ }^{2}$ RisingSun Market Research \& Consulting, Shanghai, China

Correspondence to Dr Qinghua Nian, Institute for Global Tobacco Control, Department of Health, Behavior and Society, Johns Hopkins University Bloomberg School of Public Health, Baltimore, MD 21205, USA; qnian1@jhu.edu

Received 7 April 2021 Accepted 22 June 2021
A) Check for updates

(c) Author(s) (or their employer(s)) 2021. Re-use permitted under CC BY-NC. No commercial re-use. See rights and permissions. Published by BMJ.

To cite: Nian Q, Hardesty JJ, Cohen JE, et al. Tob Control Epub ahead of print: [please include Day Month Year]. doi:10.1136/

tobaccocontrol-2021-056703

\section{ABSTRACT \\ Objective This study, conducted in China, evaluated the effectiveness of four different themes of health warning labels (HWLs) that used both text and pictures: (1) self-harm from using cigarettes, (2) harming family or children with secondhand smoke, (3) reinforcing compliance with existing smoke-free policies and (4) anticigarette gift giving practices.}

Methods A cross-sectional randomised experimental survey was conducted among 3247 adult (aged 18+ years) participants in Beijing, Shanghai and Shenzhen in 2017, using quotas for age group, gender and smoking status. Participants were randomly assigned to one of the four HWL themes. Each participant viewed eight HWLS and rated how effective these themed-labels were in terms of credibility, raising awareness of health harms of smoking on family and children, improving compliance with public smoking bans, stopping the practice of gifting cigarettes, thinking about quitting and preventing smoking using a 10-point scale, with 10 being most effective. Analysis of variance and independent t-tests were used to analyse these data.

Findings All four HWL themes performed well for each outcome with average ratings $>6.5$. Harming family or children with secondhand smoke was the theme that received the highest ratings for each outcome, with credibility $(8.0,95 \% \mathrm{Cl} 7.86$ to 8.09$)$ and prevention of smoking $(8.8,95 \% \mathrm{Cl} 8.63$ to 8.91$)$ outcomes being significantly higher $(p<0.05)$. Overall, analysis of ratings by gender, income and education did not impact outcomes.

Conclusion All four HWL themes tested could be effective in China; the theme of secondhand smoke harming family or children may be a particularly credible/ effective theme.

\section{INTRODUCTION}

Pictorial health warning labels (HWLs) on cigarette packages can serve as a credible source of information about the harms of tobacco use and are more effective than text-only health warnings. ${ }^{1-4}$ At least 125 countries or jurisdictions have policies requiring pictorial HWLs on cigarette packages. ${ }^{5}$ Research conducted in countries where pictorial HWLs have been implemented, including Australia, Brazil, Canada, Mauritius, Thailand and Uruguay, has demonstrated pictorial HWLs are effective in increasing knowledge of the harms of smoking among smokers and non-smokers, supporting cessation-seeking behaviours, supporting smokers from relapsing and addressing tobacco-related disparities associated with education. ${ }^{6-16}$

In China, there are approximately 308 million cigarette smokers and 732 million non-smokers who are regularly exposed to secondhand smoke (SHS). ${ }^{17}$ Approximately 50\% of men and 2\% of women smoke tobacco currently. ${ }^{17}$ Annually, smoking-related diseases such as cancer, stroke and chronic obstructive pulmonary diseases (COPD, such as emphysema) kill 1.4 million people in China $^{18}$ and this number is predicted to rise to $>5$ million by $2050 .^{19}$ Although awareness of the harms of cigarettes has increased in China among the general population from 39\% aware smoking causing heart attack in 2010 to $51 \%$ in 2018, ${ }^{1718} 20$ most Chinese smokers lack knowledge of the major health consequences of smoking. ${ }^{8}{ }^{14}$ Results from the 2009-2010 International Tobacco Control (ITC) study reported that Chinese adult smokers had the lowest levels of knowledge regarding chronic health conditions caused by smoking among the 18 countries studied. Among a sample of adult Chinese smokers in the ITC study, 54\% correctly knew that smoking caused heart disease and $30 \%$ knew that smoking caused strokes. ${ }^{2}$ By comparison, $93 \%$ and $87 \%$ of Canadian smokers reported this knowledge, respectively. ${ }^{2}$ The inclusion of information on HWLs that describes the specific harms caused by smoking could be a costeffective measure to improve Chinese smokers' and non-smokers' knowledge and influence their perceived risk of using cigarettes. ${ }^{21}$ China has used text-only HWLs since 1991. The current Chinese HWLs include two messages-“smoking may harm your health" and "quit[ting] smoking early is good for your health". Countries where pictorial HWLs are adopted, such as Mexico and Canada, use rotations of pictorial messages to help maintain effectiveness. It is common for countries to have several pictorial messages in each rotation. In Canada, each rotation includes 16 pictorial messages. Previous studies have indicated that most Chinese smokers and non-smokers were supportive of improving HWLs on cigarettes by including additional health information. ${ }^{19} 22$

To learn more about effective features for inclusion on future pictorial HWLs in China, we designed HWLs considering common practices for pictorial HWL design in other jurisdictions. When reviewing HWLs appearing on cigarette packs across the world, the content primarily includes information about health consequences to the 
cigarette user. Some of the most common health consequences from smoking featured on HWLs are cancer and lung disease; however, some also include messages that communicate the health consequences to non-users from exposure to SHS. Family concerns are found as one of the most significant reasons for quitting among Chinese smokers which supports the view that, in general, Chinese people cherish their collectivistic values and have a strong sense of obligation and responsibility to family. ${ }^{23-27}$ We hypothesised that HWLs could be effective if they communicate information about the harms of SHS on family or children. The practices around tobacco use and tobacco control policy in China were also considered when designing the HWLs to be tested. Many Chinese cities have adopted smoke-free laws since 2008 including Beijing in 2015, Shanghai in 2017 and Shenzhen in $2017 .{ }^{28}$ Each of these cities adopted a $100 \%$ smoke-free policy as per standards outlined by WHO's Framework Convention on Tobacco Control Article 8. An HWL that reminds people about the smoke-free law could reinforce knowledge about the dangers of SHS and the collective responsibility for clean air. Additionally, we considered China-specific cultural practices around cigarettes. Cigarette giving or gifting is a tradition in China commonly practised during holidays and special occasions, such as the Mid-Autumn Festival and Chinese New Year. In 2012, one in seven families gifted cigarettes to others in the last year in Zhejiang Province. ${ }^{29}$ Gifting cigarettes is done to establish or maintain interpersonal relationships. Developing an HWL that links cigarette use to lung health could challenge this practice by highlighting how inconsistent it is to gift a product that is harmful.

Based on these considerations, four different pictorial HWL themes were developed: (1) self-harm from using cigarettes, (2) harming family or children with SHS, (3) reinforcing compliance with existing smoke-free policies and (4) anticigarette gift giving practices. The study designed eight different HWLs for each of these themes. The present study sought to measure the perceived effectiveness and credibility of each of the four designed themes, and their potential to influence behaviour. The study further explored differences in these various outcomes based on smoking status, age, gender, education and income. To the best of our knowledge, this is the first study to comprehensively evaluate cultural HWLs themes in China. This study can inform the choice of HWL themes for China when it is ready to adopt pictorial HWLs.

\section{RESEARCH METHODOLOGY}

This study used the Strengthening the Reporting of Observational Studies in Epidemiology cross-sectional reporting guidelines. ${ }^{30}$

\section{Sample and recruitment}

Data collection was performed by a Chinese data collection firm with teams located in Beijing, Shanghai and Shenzhen during 2017. A co-investigator and two graduate students from Johns Hopkins Bloomberg School of Public Health conducted in-person trainings with research firm staff.

The study collected survey data from adults (aged 18 years and older). Potential survey participants were recruited in public spaces at retail settings, such as malls. Survey participants needed to be: (1) residents of their city for at least 1 year and (2) not professionally affiliated with the tobacco industry. In each city, the sample included quotas for gender $(50 \%$ male, $50 \%$ female), age (50\% 18-39 years old, 50\% 40 years and older) and smoking status (50\% cigarette smoker, $50 \%$ non-smoker) to ensure that each analysis group had a minimum of 400 participants to achieve statistical power at $0.8(\alpha=0.05)$ for an effect size of 0.2. Male participants were defined as smokers if they reported 'Yes' to the question "Do you smoke daily?" and reported ' 100 or more cigarettes' to the question "How many cigarettes have you smoked in your life?" With consideration of the lower prevalence of current smoking among women, female participants were defined as smokers if they reported 'Yes' to the question "Have you smoked in the last 30 days?" and reported ' 100 or more cigarettes' to the question "How many cigarettes have you smoked in your life?" Otherwise, they were defined as non-smokers. Survey participants received a voucher for 50 RMB ( US\$7.37) for their time.

\section{Health warning label theme design}

The study designed HWLs that used one of four themes, including: (1) self-harm from using cigarettes, (2) harming family or children with SHS, (3) reinforcing compliance with existing smoke-free policies and (4) anticigarette gift giving practices. Each of the four HWL themes had eight different HWLs designs. Warning label images were paired with a primary message, that is, 'Smoking causes lung cancer' or 'Smoking causes lung disease', secondary message, for example, "Let your lungs heal: Quitting today may improve your coughing within a few months", and a quit message, that is, 'Thinking about quitting? Call 400-8085531'. The primary messages were intended to inform the participants about the health effects of smoking ('self-harm from using cigarettes' and 'anticigarette gift giving practices') and the effects of SHS on others ('harming family or children with SHS' and 'reinforcing compliance with existing smoke-free policies'). Among the eight HWLs in both 'self-harm from using cigarettes' and 'anticigarette gift giving practices' themes, four used the primary message 'Smoking causes lung cancer' and four used 'Smoking causes lung disease'. Similarly, the 'harming family or children with SHS' and 'reinforcing compliance with existing smoke-free policies' themes used the following primary messages: 'Secondhand smoke causes lung cancer' and 'Secondhand smoke causes asthma', with each primary message equally split among the eight HWLs for both themes. To minimise confounding by health effect, the text focused on lung health across all four themes. Secondary messages provided additional factual and motivational messages that may further support quit attempts and were necessary to further develop thematic messages. The number of unique secondary messages varied by theme. Among the 'self-harm from using cigarettes', 'harming family or children with SHS', 'reinforcing compliance with existing smokefree policies' and 'anticigarette gift giving practices' themes, there were seven, six, four and seven unique messages, respectively. To get to eight unique HWLs, secondary messages were repeated with the alternative primary message. One quit message was used across all 32 HWLs: 'Thinking about quitting? Call 400-808-5531'. One of the eight HWLs from each theme is highlighted in figure 1. All 32 designed HWLs can be found in online supplemental appendix figures A1-A4. Participants were randomly assigned to one of the four themes and viewed all eight HWLs corresponding to the theme, one at a time. Participants evaluated the effectiveness of the HWLs by answering the seven rating questions (described in the 'Measures' section).

\section{Measures}

Effectiveness of themed HWLs was measured on a scale of 1 to $10(1=$ 'not at all', $10=$ 'extremely') for whether the themed label (1) was credible, (2) might prevent the gifting of cigarettes, (3) made people concerned about smoking near their family, (4) 


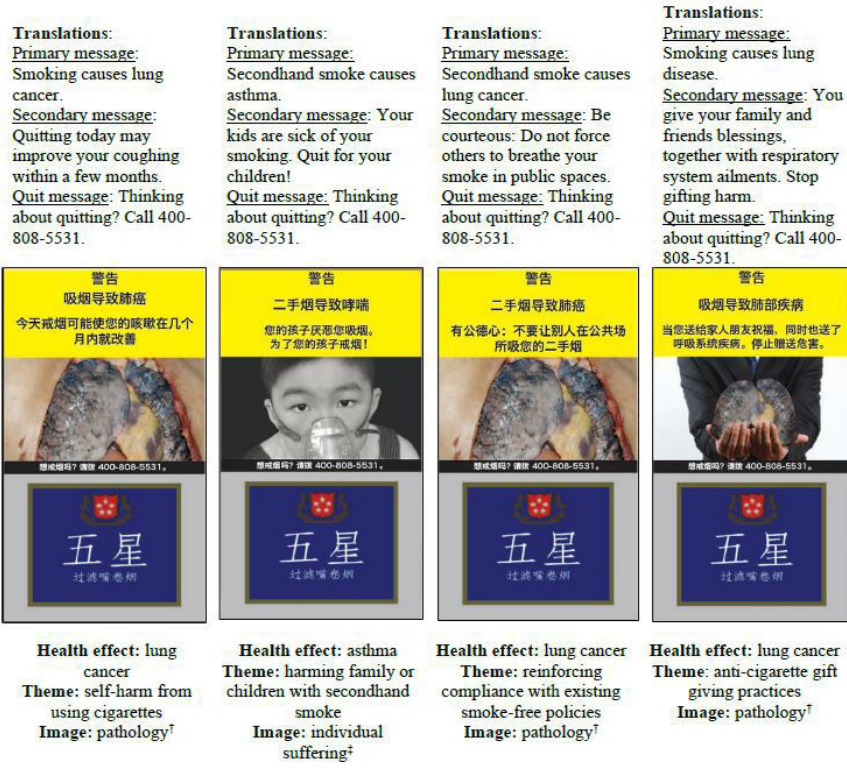

Figure 1 Sample of pictorial health warning labels (HWLs). Each HWL in the figure represents one of the eight HWLs from each theme. Online supplemental figures include all $32 \mathrm{HWLs}$ viewed. +Courtesy of Dr Prakit, Action on Smoking and Health Foundation, Thailand. ¥Her Majesty the Queen in Right of Canada, represented by the Minister of Health (2013).

made people concerned about smoking near children, (5) made people more likely to comply with public smoking bans, (6) made people think about quitting and (7) might prevent people from smoking.

Four sociodemographic characteristics were dichotomised for the analysis: gender (male, female); age ( $<40, \geq 40$ years); annual household income ( $\leq 100000 \mathrm{RMB},>100000 \mathrm{RMB}$ ) and educational attainment (<college degree, college degree).

\section{Statistical analysis}

Statistical analyses were performed using SPSS for Windows, V.27.0 (SPSS, Chicago, Illinois, USA). Descriptive statistics, including means and SD or frequency distributions, were used to describe the study sample and variables. Analysis of variance models were performed to analyse the differences in rating scores in relation to themes of HWL. Tukey's honest significant difference test was used as post hoc test to detect significant differences in rating scores for the seven outcomes between the four HWL themes. In addition, independent t-tests were used to investigate the differences between groups with various sociodemographic characteristics when evaluating the effectiveness of HWL themes. Complete case analysis was used to deal with missing data. We excluded participants with at least one missing value for any of the variables used in the analysis $(0.49 \%$ of the total participants). For each analysis, a two-sided $\mathrm{p}<0.05$ was used to determine statistical significance.

\section{RESULTS}

\section{Sample}

In total, there were $n=3247$ participants with approximately equal distribution between the three cities (Beijing, $n=1090$, Shanghai $n=1082$, Shenzhen $n=1075$ ). Significantly lower percentages of participants from Shenzhen had a college degree or higher $(31.7 \%)$ or earned an annual household income $>100$ 000 RMB (40.9\%) than participants from Beijing $(54.5 \%$ and

\begin{tabular}{|c|c|c|c|c|}
\hline & $\begin{array}{l}\text { Smoker } \\
(n=1622)\end{array}$ & $\begin{array}{l}\text { Non-smoker } \\
(n=1625)\end{array}$ & $\begin{array}{l}\text { Total } \\
(\mathrm{n}=3247)\end{array}$ & $P$ value* \\
\hline \multicolumn{5}{|l|}{ Age (years) } \\
\hline$<40$ & $50.1 \%$ & $50.0 \%$ & $50.0 \%$ & \multirow[t]{2}{*}{0.986} \\
\hline$\geq 40$ & $49.9 \%$ & $50.0 \%$ & $50.0 \%$ & \\
\hline \multicolumn{5}{|l|}{ Gender } \\
\hline Male & $50.1 \%$ & $50.1 \%$ & $50.1 \%$ & \multirow[t]{2}{*}{0.986} \\
\hline Female & $49.9 \%$ & $49.9 \%$ & $49.9 \%$ & \\
\hline \multicolumn{5}{|l|}{$\begin{array}{l}\text { Annual household income } \\
\text { (RMB) }\end{array}$} \\
\hline$\leq 100000$ & $48.5 \%$ & $48.7 \%$ & $48.6 \%$ & \multirow[t]{3}{*}{0.860} \\
\hline$>100000$ & $51.4 \%$ & $51.0 \%$ & $51.2 \%$ & \\
\hline Missing & $0.1 \%$ & $0.3 \%$ & $0.2 \%$ & \\
\hline \multicolumn{5}{|l|}{ Education } \\
\hline $\begin{array}{l}\text { Primary school or less } \\
\text { (grades 1-6 or less) }\end{array}$ & $3.0 \%$ & $3.7 \%$ & $3.3 \%$ & \multirow[t]{5}{*}{0.301} \\
\hline $\begin{array}{l}\text { Secondary school } \\
\text { (grades 7-9) }\end{array}$ & $13.1 \%$ & $12.8 \%$ & $12.9 \%$ & \\
\hline $\begin{array}{l}\text { High school or } \\
\text { vocational school } \\
\text { (grades 10-12) }\end{array}$ & $37.1 \%$ & $34.5 \%$ & $35.8 \%$ & \\
\hline College or higher & $46.6 \%$ & $48.7 \%$ & $47.7 \%$ & \\
\hline Missing & $0.3 \%$ & $0.3 \%$ & $0.3 \%$ & \\
\hline
\end{tabular}

${ }^{*} \mathrm{P}$ value of $\chi^{2}$ tests.

48.9\%, respectively) and Shanghai $(57.0 \%$ and $63.9 \%$, respectively) $(\mathrm{p}<0.001)$. Smokers and non-smokers in this study had similar sociodemographic characteristics (table 1). About onehalf $(48.6 \%)$ had an annual household income of $<100000$ $\mathrm{RMB}$, and $47.7 \%$ had a college degree or higher.

\section{HWL effectiveness by HWL theme}

Both smokers and non-smokers had the opportunity to assess the effectiveness of HWL themes with regard to their credibility and anticigarette gift giving. Only smokers were asked to assess the effectiveness of HWL themes with regard to quitting, concerns about smoking near their family and children and compliance with public smoking bans; and only non-smokers were asked to assess the effectiveness of HWL themes with regard to smoking prevention. For each of the seven outcomes, all four HWL themes received rating scores $>6.5$ from smokers and $>8.0$ from non-smokers (table 2). Among the four HWL themes, the theme of SHS harming family or children was rated the highest.

There were significant differences across the four HWL themes in mean effectiveness with regard to credibility among smokers, $\mathrm{F}(3,1612)=4.50, \mathrm{p}=0.004$ and non-smokers, $\mathrm{F}(3,1611)=2.83$, $\mathrm{p}=0.037$; and potential to prevent people from smoking, $\mathrm{F}(3,1611)=3.35, \mathrm{p}=0.018$. In terms of credibility, SHS harming family or children themed HWLs performed $6 \%$ better than self-harm among smokers $(p=0.007)$. It was also found that harming family or children themed HWLs performed $5 \%$ and $4 \%$ better than anticigarette gift giving themed HWLs among smokers $(p=0.011)$ and non-smokers $(p=0.028)$, respectively. In response to the smoking prevention outcome, harming family or children themed HWLs performed 4\% better than anticigarette gift giving themed HWLs $(p=0.009)$. No significant differences were found between self-harm, smoke-free and anticigarette gift giving themes with respect to each of the seven outcomes. 
Table 2 Ratings of effectiveness of HWL themes, by smoking status

\begin{tabular}{|c|c|c|c|c|c|}
\hline Outcomes & $\begin{array}{l}\text { Self-harm from using } \\
\text { cigarettes }\end{array}$ & $\begin{array}{l}\text { Harming family /children } \\
\text { with SHS }\end{array}$ & $\begin{array}{l}\text { Reinforcing compliance with } \\
\text { existing smoke-free policies }\end{array}$ & $\begin{array}{l}\text { Anticigarette gift } \\
\text { giving }\end{array}$ & \\
\hline$(1-10)$ & $\left(n_{1}=394 ; n_{2}=403\right)$ & $\left(n_{1}=406 ; n_{2}=409\right)$ & $\left(n_{1}=392 ; n_{2}=386\right)$ & $\left(n_{1}=424 ; n_{2}=417\right)$ & $P$ value* \\
\hline \multicolumn{6}{|l|}{ The warning label is credible } \\
\hline Smokers: mean (SD) & $6.99(1.71)$ & $7.40(1.75)$ & $7.10(1.81)$ & $7.02(1.77)$ & 0.004 \\
\hline Non-smokers: mean (SD) & $8.33(1.43)$ & $8.56(1.41)$ & $8.37(1.50)$ & $8.27(1.56)$ & 0.037 \\
\hline \multicolumn{6}{|c|}{ The warning label would stop me from gifting cigarettes } \\
\hline Smokers: mean (SD) & $6.82(2.03)$ & $7.04(1.98)$ & $6.73(1.99)$ & $6.84(2.05)$ & 0.161 \\
\hline Non-smokers: mean (SD) & $8.27(1.59)$ & $8.45(1.64)$ & $8.37(1.53)$ & $8.26(1.68)$ & 0.298 \\
\hline \multicolumn{6}{|c|}{ The warning label makes me think about quitting } \\
\hline Smokers: mean (SD) & $6.73(1.95)$ & $6.94(2.03)$ & $6.72(2.00)$ & $6.69(2.05)$ & 0.274 \\
\hline \multicolumn{6}{|c|}{ The warning label makes me concerned about smoking near my family } \\
\hline Smokers: mean (SD) & $7.16(1.81)$ & $7.37(1.78)$ & $7.17(1.82)$ & $7.08(1.92)$ & 0.137 \\
\hline \multicolumn{6}{|c|}{ The warning label makes me concerned about smoking near children } \\
\hline Smokers: mean (SD) & $7.46(1.82)$ & $7.67(1.76)$ & $7.45(1.84)$ & $7.33(1.89)$ & 0.060 \\
\hline \multicolumn{6}{|c|}{ The warning label makes me more likely to comply with public smoking bans } \\
\hline Smokers: mean (SD) & $7.21(1.90)$ & $7.43(1.86)$ & $7.33(1.84)$ & $7.16(1.98)$ & 0.184 \\
\hline \multicolumn{6}{|c|}{ The warning label will prevent me from smoking } \\
\hline Non-smokers: mean (SD) & $8.61(1.47)$ & $8.78(1.45)$ & $8.65(1.51)$ & $8.44(1.70)$ & 0.018 \\
\hline
\end{tabular}

$\mathrm{n}_{1}$, number of smokers; $\mathrm{n}_{2}$, number of non-smokers.

* $P$ value of analysis of variance.

HWL, health warning label; SHS, secondhand smoke.

\section{HWL effectiveness by sociodemographic characteristics and smoking status}

All smoker and non-smoker subgroups, by age, gender, household annual income and education level, rated the SHS harming family or children theme the highest for each outcome. The differences between smoker subgroups with various sociodemographic characteristics in assessing the effectiveness of HWL themes were not statistically significant. However, non-smokers aged 40 years older, and non-smokers with annual household incomes of $>100000 \mathrm{RMB}$, were more likely to rate all HWL themes higher than their younger or lower-income peers for the smoking prevention outcome $(\mathrm{p}=0.009$ and $\mathrm{p}<0.001$, respectively). In addition, it was found that non-smokers rated all four HWL themes higher than smokers with regard to their credibility $(\mathrm{p}<0.001)$ and potential to prevent the gifting of cigarettes $(\mathrm{p}<0.001)$.

\section{DISCUSSION}

With a focus on China-specific culture and tobacco control policies, this study designed four pictorial HWL themes that conveyed various harms of smoking and SHS and evaluated their effectiveness. Overall, all HWL themes tested in this study performed well in their credibility and potential to elicit positive behaviour outcomes, and SHS harming family or children theme performed the best among all themes tested. There were minimal differences based on sociodemographic characteristics among smokers in assessing the effectiveness of HWL themes. However, smoking status was relevant in terms of ratings with generally higher ratings among non-smokers than smokers.

China has the largest smoking population worldwide and Chinese smokers' knowledge of health effects of smoking is relatively low. Designing thematic pictorial HWLs like those developed for this study could be helpful in addressing low knowledge of smoking harms, influencing perceived risk, supporting cessation-seeking behaviours, discouraging cigarette gift giving and preventing smoking initiation in China. As a result of their repeated exposure, wide reach and low cost, pictorial HWLs are one of the best measures available to governments to educate people about harms of tobacco products. By employing images and text, pictorial HWLs can depict a broad range of specific health harms that are caused by smoking, including lung cancer, heart disease, stroke, mouth disease, throat cancer, blindness, COPD, peripheral vascular disease and impotence. ${ }^{31}$ Nonetheless, limited work has been done to characterise the potential impacts of SHS on family or community on warning labels. Cultural norms and social environment of a country may play a role in the effectiveness of HWL design in Asian countries. ${ }^{32}$ Research examining ways to better implement HWLs on tobacco have highlighted the need to consider culture as well as knowledge of health effects from smoking to better tailor label content. ${ }^{33}$ However, experimental studies testing perceptions of health warnings among adult smokers and youth in seven different countries (including China) concluded that there is efficacy of HWLs across cultural contexts. ${ }^{34}$ Chinese culture tends to be more collectivistic and emphasises the needs and desires of the family over the individual. ${ }^{23}$ This may explain why exposure to the 'SHS harming family or children' HWL theme performed consistently better than the other themes and significantly better than the others in terms of credibility and smoking prevention in this study. Others have found that Chinese smokers reported concerns for close friends and relatives as a reason to quit smoking. ${ }^{35}$ Smokers reported that the effect of cigarette smoke on non-smokers (45\%), family disapproval (42\%) and concern for setting an example for children (41\%) were reasons for thinking about quitting. These findings are consistent with a collectivistic culture in China. ${ }^{35}$ Several studies indicate there are potential benefits to incorporating collectivistic values when designing tobacco control interventions. ${ }^{23} 2536$ By raising people's concerns about the threat of smoking to their family and children, interventions addressing collectivistic values may be more likely to increase interest in quitting. Other studies indicate that some Chinese parents do not know about the harms of SHS exposure on children. ${ }^{37}$ HWLs that depict the harms of SHS on family or children may inform smokers about the health 
consequences of smoking on their family and children, promote smoke-free homes and increase their intentions to quit smoking.

The theme reinforcing compliance with existing smoke-free policies was found to be as effective as the theme that communicated individual harms and this theme could be a viable option for China. Currently, there is no national smoke-free law and over half of the adopted subnational laws in China do not ban all indoor smoking and still allow designated indoor smoking rooms. ${ }^{28}$ Many cities experience challenges in enforcing smokefree laws due to resource constraints. ${ }^{28} 38$ An effective HWL theme that reinforces compliance with existing smoke-free policies, such as the one tested in this study, can serve as a costeffective measure to inform people about the harms of SHS and may encourage people to comply with public smoking bans. One important caveat is that HWL laws are national and smoke-free laws in China have been subnational to date; therefore, HWLs with a smoke-free law compliance theme should only be implemented if a corresponding national smoke-free law is in place.

Gifting cigarettes is a medium for establishing and maintaining social relationships in Chinese culture. ${ }^{39-42}$ Cigarette gift giving promotes the social acceptability and desirability of smoking and contributes to smoking initiation and failure to quit. ${ }^{43}$ The current text-only Chinese warning labels do not address the harms of the cigarette gift giving culture and have a limited impact on gifting cigarettes. ${ }^{44}$ This study demonstrated that anticigarette gifting themed HWLs can be as effective as other themes in trustworthiness and may have potential to prevent the gifting of cigarettes, make people concerned about smoking near their family or children, more likely to comply with public smoking bans and evoke intention to quit smoking. Using the anticigarette gifting HWL theme may denormalise smoking by changing social norms around the Chinese tradition of gifting cigarettes.

This study has several limitations. First, the research was conducted in three cities with high development and are not fully representative of the geographical region and economic development in China. The findings therefore may not be generalised to the Chinese population living in underdeveloped rural areas or smaller, less developed cities. Also, because of the convenience sampling, the generalisability of the findings to smokers and non-smokers in China is unclear. This study considered male participants as smokers only if they reported smoking daily (44\% of current male smokers smoke daily according to 2018 China Global Adult Tobacco Survey) ${ }^{17}$; thus, the findings regarding male smokers may not be generalisable to non-daily male smokers in China. In addition, the rating measures used were self-reported which are subject to demand characteristics and social desirability response bias. Another important limitation to note is around our measurement of gender. In Chinese, the same word is used for both 'sex' and 'gender', and therefore, respondents may have answered this differently. Despite these limitations, this study provides evidence for designing pictorial HWLs in China. The tested themes were credible, effective and relevant to China-specific smoking-related behaviours and social norm changes. All four themes assessed in the study could be considered by health authorities as promising avenues to include in tobacco control efforts. This study can inform HWL policies in China that can ultimately have a substantial impact on the health of Chinese citizens.

\section{CONCLUSION}

Given the high rating scores of all tested themes, the self-harm from using cigarettes, harming family or children from SHS, reinforcing compliance with existing smoke-free policies and anticigarette gift giving practices themed HWLs may all be efficacious in raising the awareness of smoking harms and may elicit positive behaviour outcomes. The harming family or children theme was more credible and more effective with regard to the smoking prevention outcome than other themes and may be the most compelling theme for use in China. These findings have important implications for informing the design of pictorial HWLs. They may address China-specific culture and challenges, better educate the public about individual and collective harms of tobacco products and alter social norms regarding smoking in China. It is also possible they may help prevent never and former smokers from initiating cigarette use and encourage current smokers to plan to quit or think about quitting. In addition, smokers may become more concerned about smoking near family and children and more likely to comply with smoke-free laws. Smokers and non-smokers may also be less likely to gift cigarettes.

\section{What this paper adds}

\section{What is already known on this subject}

- Pictorial health warning labels (HWLs) on cigarette packaging can better communicate credible information about health harms of tobacco use among smokers and non-smokers and support cessation-seeking behaviours than text-only health warnings.

- China has HWLs that only use text.

- Most Chinese smokers are not as aware as smokers in other countries about the health risks of smoking.

What important gaps in knowledge exist on this topic

- Limited work has been done to develop and test different HWL themes/content in China.

- In designing HWLs to be tested, we considered common practices for pictorial HWL design in other jurisdictions, practices around tobacco use and tobacco control policy in China and China-specific culture.

What this paper adds

- This study develops and assesses four HWL themes (ie, selfharm from using cigarettes, harming family or children with secondhand smoke, reinforcing compliance with existing smoke-free policies and anticigarette gift giving practices) and finds that all four tested themes could be efficacious in China; the theme of harming family or children theme may be a particularly credible theme and more effective in eliciting smoking prevention outcomes.

Twitter Jeffrey J Hardesty @HardestyJeffrey and Ryan David Kennedy @com/ ryan_david

Contributors QN, JJH, RDK and JEC conceived the study. RDK, JJH and XX performed the data collection. QN performed the analysis and drafted the manuscript. All the authors contributed to the interpretation of data and provided critical feedback on the drafts of the manuscript; they also approved the final version of the manuscript.

Funding This work was supported with funding from Bloomberg Philanthropies' Bloomberg Initiative to Reduce Tobacco Use (http://www.bloomberg.org/).

Competing interests None declared.

Patient consent for publication Not required.

Ethics approval The study received an exemption determination from the Institutional Review Board of the Johns Hopkins Bloomberg School of Public Health.

Provenance and peer review Not commissioned; externally peer reviewed. 
Data availability statement Data are available on reasonable request.

Open access This is an open access article distributed in accordance with the Creative Commons Attribution Non Commercial (CC BY-NC 4.0) license, which permits others to distribute, remix, adapt, build upon this work non-commercially, and license their derivative works on different terms, provided the original work is properly cited, appropriate credit is given, any changes made indicated, and the use is non-commercial. See: http://creativecommons.org/licenses/by-nc/4.0/.

\section{ORCID iDs}

Qinghua Nian http://orcid.org/0000-0001-9551-1599

Jeffrey J Hardesty http://orcid.org/0000-0002-4705-4976

Joanna E Cohen http://orcid.org/0000-0002-3869-3637

\section{REFERENCES}

1 Hammond D, Fong GT, McNeill A, et al. Effectiveness of cigarette warning labels in informing smokers about the risks of smoking: findings from the International tobacco control (ITC) four country survey. Tob Control 2006;15(Suppl 3):iii19-25.

2 International Tobacco Control Project. Health warnings on tobacco packages: ITC cross-country comparison report, 2012. Available: http://www.itcproject.org/files/lTC_ Cross-Country_Report_-_Warning_Labels-Final.pdf Chinese: http://www.itcproject. org/files/ITC_Cross-Country-Labelchinese-May18v3-web.pdf

3 Hammond D. Health warning messages on tobacco products: a review. Tob Control 2011;20:327-37.

4 Fong GT, Hammond D, Hitchman SC. The impact of pictures on the effectiveness of tobacco warnings. Bull World Health Organ 2009;87:640-3.

5 Campaign for tobacco-free kids legal website, 2020. Available: www. tobaccocontrollaws.org

6 Yong $\mathrm{H}-\mathrm{H}$, Fong GT, Driezen $\mathrm{P}$, et al. Adult smokers' reactions to pictorial health warning labels on cigarette packs in Thailand and moderating effects of type of cigarette smoked: findings from the International tobacco control Southeast Asia survey. Nicotine Tob Res 2013;15:1339-47.

7 Fathelrahman Al, Omar M, Awang R, et al. Smokers' responses toward cigarette pack warning labels in predicting quit intention, stage of change, and self-efficacy. Nicotine Tob Res 2009;11:248-53.

8 ITC Project, World Health Organization, and World Heart Federation. Cardiovascular harms from tobacco use and secondhand smoke: global gaps in awareness and implications for action. Waterloo, Ontario, Canada and Geneva, Switzerland, Apr 2012. Available: http://www.who.int/tobacco/publications/surveillance/ cardiovascular_harms_tobacco_use/en

9 Gravely S, Fong GT, Driezen P, et al. The impact of the 2009/2010 enhancement of cigarette health warning labels in Uruguay: longitudinal findings from the International tobacco control (ITC) Uruguay survey. Tob Control 2016;25:89-95.

10 Green AC, Kaai SC, Fong GT, et al. Investigating the effectiveness of pictorial health warnings in Mauritius: findings from the ITC Mauritius survey. Nicotine Tob Res 2014;16:1240-7.

11 Thrasher JF, Villalobos V, Szklo A, et al. Assessing the impact of cigarette package health warning labels: a cross-country comparison in Brazil, Uruguay and Mexico. Salud Publica Mex 2010;52 (Suppl 2):S206-15.

12 Borland $\mathrm{R}$, Yong $\mathrm{H}-\mathrm{H}$, Wilson $\mathrm{N}$, et al. How reactions to cigarette packet health warnings influence quitting: findings from the ITC four-country survey. Addiction 2009;104:669-75.

13 Huang J, Chaloupka FJ, Fong GT. Cigarette graphic warning labels and smoking prevalence in Canada: a critical examination and reformulation of the FDA regulatory impact analysis. Tob Control 2014;23 (Suppl 1):i7-12.

14 World Health Organization Western Pacific Region, University of Waterloo, ITC Project, ThinkTank Research Center for Health Development. Tobacco health warnings in China: evidence of effectiveness and implications for action, 2014. Available: https:// www.openaire.eu/search/publication?articleld=dedup_wf_001::d89841e2e93f4dde b80f9bd98c4d3536

15 Hammond D, Fong GT, McDonald PW, et al. Graphic Canadian cigarette warning labels and adverse outcomes: evidence from Canadian smokers. Am J Public Health 2004;94:1442-5.

16 Li J, Grigg M. New Zealand: new graphic warnings encourage registrations with the Quitline. Tob Control 2009;18:72.

17 Chinese Center for Disease Control and Prevention. Global adult tobacco survey: 2018 China report. Beijing, China. Three Gorges Publishing House, 2018. https:// extranet.who.int/ncdsmicrodata/index.php/catalog/803

18 Yang G, Wang Y, Wu Y, et al. The road to effective tobacco control in China. Lancet 2015:385:1019-28.
19 Elton-Marshall T, Xu SS, Meng G, et al. The lower effectiveness of text-only health warnings in China compared to pictorial health warnings in Malaysia. Tob Control 2015;24 (Suppl 4):iv6-13.

20 Chinese Center for Disease Control and Prevention. 2010 China global adult tobacco survey report. Available: https://extranet.who.int/ncdsmicrodata/index.php/catalog/ 252/related_materials

21 Yang J, Hammond D, Driezen P, et al. Health knowledge and perception of risks among Chinese smokers and non-smokers: findings from the wave 1 ITC China survey. Tob Control 2010;19 (Suppl 2):i18-23.

22 Fong GT, Hammond D, Jiang Y, et al. Perceptions of tobacco health warnings in China compared with picture and text-only health warnings from other countries: an experimental study. Tob Control 2010;19 (Suppl 2):i69-77.

23 Yang T, Fisher KJ, Li F, et al. Attitudes to smoking cessation and triggers to relapse among Chinese male smokers. BMC Public Health 2006:6:65-7.

24 Madewell ZJ. The belief that secondhand smoke causes serious illness among Chinese smokers: smoking cessation and intention to quit. Tob Prev Cessat 2018;4:5.

25 Shakib S, Zheng $\mathrm{H}$, Johnson CA, et al. Family characteristics and smoking among urban and rural adolescents living in China. Prev Med 2005;40:83-91.

26 Unger JB, Yan L, Shakib S, et al. Peer influences and access to cigarettes as correlates of adolescent smoking: a cross-cultural comparison of Wuhan, China, and California. Prev Med 2002;34:476-84.

27 Trinidad DR, Chou C-P, Unger JB, et al. Family harmony as a protective factor against adolescent tobacco and alcohol use in Wuhan, China. Subst Use Misuse 2003;38:1159-71.

28 Lin H, Chang C, Liu Z, et al. Subnational smoke-free laws in China. Tob Induc Dis 2019;17:78.

$29 \mathrm{Xu}$ Y, Xu S, Wu Q, et al. Association between secondhand smoke exposure at home and cigarette gifting and sharing in Zhejiang, China: a repeat cross-sectional study. BMJ Open 2016;6:e010058.

30 von Elm E, Altman DG, Egger $M$, et al. The strengthening the reporting of observational studies in epidemiology (STROBE) statement: guidelines for reporting observational studies. J Clin Epidemiol 2008;61:344-9.

31 Cunningham R. Gruesome photos on cigarette packages reduce tobacco use. Bull World Health Organ 2009;87:569.

32 Reid JL, Mutti-Packer S, Gupta PC, et al. Influence of health warnings on beliefs about the health effects of cigarette smoking, in the context of an experimental study in four Asian countries. Int J Environ Res Public Health 2017;14:868.

33 Ratneswaran C, Chisnall B, Li M, et al. Desensitisation to cigarette package graphic health warnings: a cohort comparison between London and Singapore. BMJ Open 2016;6:10.

34 Hammond D, Reid JL, Driezen P, et al. Are the same health warnings effective across different countries? An experimental study in seven countries. Nicotine Tob Res 2019;21:887-95

35 Jiang Y, Elton-Marshall T, Fong GT, et al. Quitting smoking in China: findings from the ITC China survey. Tob Control 2010;19 (Suppl 2):i12-17.

36 Yang T, Miao B. Evaluation of the smoking cessation program of "Wives' help. Chinese Social Medicine 1993:47-8.

37 La Torre G, Ferketich A, Grassi MC. Tobacco smoking: the evidence from prevention and cessation. Biomed Res Int 2014;2014:1-2.

38 Nagelhout GE, de Vries H, Boudreau C, et al. Comparative impact of smoke-free legislation on smoking cessation in three European countries. Eur J Public Health 2012;22 (Suppl 1):4-9.

39 Chu A, Jiang N, Glantz SA. Transnational tobacco industry promotion of the cigarette gifting custom in China. Tob Control 2011;20:e3.

40 Liao Y, Tang J, McNeill A, et al. Impact of cigarette package warnings on attitudes towards sharing and gifting cigarettes in China: a nationwide study of smokers and non-smokers. Tob Control 2021. doi:10.1136/tobaccocontrol-2020-056160. [Epub ahead of print: 12 Jan 2021].

41 Trimble DG, Yu L, Welding K, et al. Analysis of wedding appeals on cigarette packs in China. Tob Control 2021. doi:10.1136/tobaccocontrol-2020-056189. [Epub ahead of print: 09 Apr 2021].

42 Huang L-L, Thrasher JF, Jiang Y, et al. Impact of the 'Giving Cigarettes is Giving Harm' campaign on knowledge and attitudes of Chinese smokers. Tob Control 2015;24 (Suppl 4):iv28-34.

43 Rich ZC, Xiao S. Tobacco as a social currency: cigarette gifting and sharing in China. Nicotine Tob Res 2012;14:258-63.

44 Qin Y, Wu M, Pan X, et al. Reactions of Chinese adults to warning labels on cigarette packages: a survey in Jiangsu Province. BMC Public Health 2011;11:133-40. 